Gaia Tomazzoli

\title{
Funzioni delle metafore nelle epistole arrighiane
}

\begin{abstract}
L'articolo esamina il linguaggio figurato delle epistole arrighiane (VVI-VII), con particolare attenzione alla dialettica tra oscurità e chiarezza. Per dimostrare che Dante rifiuta una poetica dell'obscuritas si propone prima un breve confronto con due tradizioni di testi il cui linguaggio è stato spesso ritenuto oscuro - quelli pseudo-profetici altomedievali e quelli riconducibili all'ars dictaminis. Si riassumono inoltre le prescrizioni dei trattati di ars dictaminis in merito ai tropi principali (pronominatio, permutatio, translatio), per poi descrivere come tali figure siano adoperate nelle epistole dantesche in ottemperanza a una retorica dell'explanatio. Si argomenta infine che tali strategie sono coerenti con l'epistemologia profetica che Dante dichiara di abbracciare in queste epistole.
\end{abstract}

The article investigates figurative language in Dante's epistles concerning Henry VII (Ep. VI-VI-VII), with particular reference to the opposition between obscurity and clarity. In order to show that Dante rejects a poetics based on obscuritas, I firstly compare these letters with two textual traditions whose language was often considered obscure, namely late Medieval pseudo-prophecies and dictamina. Secondly, I summarize the norms pertaining to the main tropes (pronominatio, permutatio, translatio) developed by the artes dictaminis, so as to show how said tropes are used, in Dante's epistles, within the frame of a rhetoric aimed at explanatio. Finally, I suggest that such strategies are consistent with the prophetical epistemology that Dante embraces in these letters.

Parole chiave: metafora, profetismo, retorica, ars dictaminis, oscurità.

«Se non fosse la nobile gagliardia dei concetti che tratto tratto lampeggiano e sfavillano attraverso l'involucro crasso o nebuloso che li ravvolge, noi non riconosceremmo agevolmente nel dettato enfatico e pesante delle Epistole l'in-

Annotazione: Ringrazio Benoît Grévin, Antonio Montefusco e Sylvain Piron per i preziosi consigli che mi hanno aiutata nella scrittura di questo articolo.

Gaia Tomazzoli, Università Ca’ Foscari Venezia

D Open Access. (C) 2020 Gaia Tomazzoli, published by De Gruyter. (c) BY This work is licensed under the Creative Commons Attribution 4.0 International License (CC BY 4.0). 
telletto sovrano [di Dante]». ${ }^{1}$ Questa ingenerosa definizione di Novati ben introduce gli snodi e i termini della questione che sarà affrontata nelle prossime pagine: la forma linguistica e retorica della prosa latina di Dante, che all'altezza del primo Novecento era pervicacemente separata dal contenuto e bollata con insofferenza come manieristica, è stata negli ultimi decenni rivalutata non solo a livello di risultati estetici, ma anche e soprattutto in relazione a un preciso codice di valori. Gli studi sull'ars dictaminis ci hanno svelato un Dante capace di maneggiare con perizia gli strumenti della tecnica epistolare in forza in quegli anni; tale tecnica è uno degli stratagemmi di legittimazione intellettuale di un messaggio cui si voleva conferire la massima importanza e sacralità. Nondimeno, la definizione di Novati chiama in causa alcuni termini fondamentali delle teorie estetiche e retoriche del Medioevo: specialmente sul piano normativo, il linguaggio era spesso concepito come involucro del contenuto, da mantenere chiaro e comprensibile. Condannata dalle prescrizioni della trattatistica, l'oscurità era rivalutata e celebrata in chiave esegetica sulla scia di Agostino: questo ambivalente rapporto produsse, lungo tutto il Medioevo, una poesia spesso incline all'enigma o all'allegoria. ${ }^{2}$

Uno dei principali strumenti di tale poetica dell'oscurità è la metafora, «chiave di volta» della retorica duecentesca - secondo una formula di Grévin ${ }^{3}$ e tratto saliente delle epistole composte da Dante per la discesa in Italia di Enrico VII. Leggendo questi testi saltano subito all'occhio, infatti, le numerose citazioni e il complesso e ricco apparato figurativo dispiegato. Poiché a tal proposito l'intertestualità è già stata ampiamente esplorata dalla critica, che ha messo in rilievo la preponderanza dell'ipotesto biblico, ${ }^{4} \mathrm{mi}$ concentrerò sulle funzioni del linguaggio figurato dantesco per coglierne la specificità dapprima attraverso un'analisi contrastiva con i principali filoni metaforici coevi e con le prescrizioni retoriche, e poi tramite un esame delle strategie discorsive delle epistole arrighiane. Il punto di partenza è dunque la constatazione che in questi documenti Dante impiega moltissime metafore e in maniera varia, immaginosa e articolata; sul significante tende a prevalere il significato, le figure di pensiero puntano

1 Novati, Le epistole, p. 6.

2 L'estetica medievale dell'obscuritas è stata oggetto di un certo numero di studi; si vedano almeno Ziolkowski, Theories of obscurity; Mehtonen, Obscure language; Sluiter, Obscurity. Sull'oscurità nella lirica italiana del Duecento si concentra il recentissimo Borsa, «Scuro saccio».

3 Grévin, Métaphore et vérité.

4 Cfr. soprattutto Rigo, Tempo liturgico; Pertile, Dante looks forward; Cura Curà, Cultura classi$c a$; Brilli, Reminiscenze scritturali. In questo stesso volume ulteriori tasselli sono aggiunti dall'articolo di Anna Fontes Baratto e, per l'epistola XI, da quelli di Rodney Lokaj e Gian Luca Potestà. 
piuttosto al procedimento analogico della metafora che non alla semplice identificazione del simbolo e sono iscritte all'interno di una precisa strategia di recupero e razionalizzazione di stilemi e immagini della tradizione; come ha rilevato Baglio, insomma, l'apparato erudito di richiami e riscritture della pagina sacra «non ha funzione meramente esornativa, poiché costituisce la consapevole e privilegiata modalità di espressione dei contenuti». ${ }^{5}$

Se muoviamo alla ricerca di un precedente per il linguaggio e per lo stile delle epistole arrighiane, le due tradizioni che sembrano manifestare una maggior prossimità con il dettato dantesco sono l'ars dictaminis e il profetismo biblico e medievale. ${ }^{6}$ Entrambe queste tradizioni - che per di più si erano spesso intrecciate, lungo il Duecento, nel corso dell'accesa disputa tra Federico II e il papato - sono caratterizzate da una prosa densa di metafore e simboli. Ragioni e caratteristiche dell'oscura metaforica dettatoria e di quella profetica sono però piuttosto differenti; la prosa epistolare dantesca sembra derivare da ciascuna delle due correnti alcuni elementi, sapientemente sistematizzati e motivati in vista di un superamento dell'oscurità.

La storia delle teorie metaforiche classiche e medievali è quella di un continuo bilanciamento tra opposte concezioni del linguaggio. Da un lato la direttrice platonica ne esalta la carica figurativa, accentuando la componente sacrale e misterica della parola: ogni atto verbale è simile a un velo che copre realtà nascoste, e il simbolo è l'unica possibile espressione di una realtà più profonda rispetto alla superficie delle cose e del discorso. L'impostazione convenzionalista del linguaggio difesa da Aristotele contro i sofisti ridimensiona invece il ruolo dell'interprete e si concentra piuttosto su un'indagine pragmatica degli effetti del linguaggio, privilegiando la retorica sull'interpretazione e la chiarezza sulla complessità. Per questa ragione lo Stagirita oppone la metafora, strumento conoscitivo e fulcro della poetica, all'enigma (Rhet. 1405a, 35-ss, Poet. 1458a, 18-24): sottratto al piano ontologico ed epistemologico e ridotto a una mera questione stilistica, l'enigma è interpretato come difetto nella tecnica poetica. ${ }^{7}$ Anche la precettistica retorica condannò per secoli l'enigma come metafora eccessivamente prolungata e oscura, ${ }^{8}$ mentre il cristianesimo neoplatonizzante e agostiniano rivendicava la scintilla ermeneutica offerta dai tropi all'interno di un'estetica della rivelazione che avvicinava teologia e poesia.

5 Ep. (Baglio), p. 13.

6 Richiama l'attenzione su questo duplice modello Brilli, The interplay, che propone anche importanti considerazioni di metodo.

7 Struck, Birth, pp. 65-6.

8 «Sed allegoria quae est obscurior "aenigma” dicitur, vitium, suo quidem iudicio, si quidem dicere dilucide virtus, quo tamen et poetae utuntur» (Quintiliano, Inst. Or. VIII, vi, 52). 


\section{L'oscurità e le immagini nel profetismo medievale}

La rivelazione contenuta nella Bibbia è innegabilmente calata in un linguaggio densamente simbolico e a tratti oscuro, il cui scopo, secondo Agostino, è molteplice: non solo scoraggiare i lettori privi di fede e di intelligenza, ma anche esaltare il piacere della lettura e rendere giustizia alla sublime natura del messaggio cristiano. ${ }^{9}$ Il discorso vale soprattutto per i passi profetici delle Scritture: ${ }^{10}$ poiché il profeta è il tramite tra l'uomo e il divino, la sua è al contempo una traduzione dal celeste all'umano e un parlare iniziatico. Dal momento che ciascun linguaggio segreto agisce tramite sostituzione, la metafora è lo strumento ideale del profeta, e perciò l'interpretazione della sua parola si realizza nella decifrazione delle immagini. ${ }^{11}$

Del resto al profeta era tradizionalmente assegnato il triplice compito di ricevere la visio, di interpretarla e di annunciarla; è soprattutto grazie alle grandi riflessioni profetologiche sviluppatesi nel XIII secolo che il momento interpretativo raggiunse la massima importanza, giusta l'autorizzazione fornita da Daniele, Aronne e Gesù, profeti il cui ruolo principale era stato quello di comprendere i segni offerti dalla realtà e i misteri disseminati nelle Scritture per poi spiegarli alla comunità. ${ }^{12}$ I problemi dell'epistemologia profetica e della comunicazione efficace potevano trovare una perfetta e comune soluzione grazie all'immagine: già Rabano Mauro paragonava la visione spirituale del profeta al linguaggio figurato che rende comprensibile ed esprimibile un concetto ineffa-

9 «Maxime autem isti docendi sunt Scripturas audire divinas, ne sordeat eis solidum eloquium, quia non est inflatum; neque arbitrentur carnalibus integumentis involuta atque operta dicta vel facta hominum, quae in illis libris leguntur, non evolvenda atque aperienda ut intelligantur, sed sic accipienda ut litterae sonant; deque ipsa utilitate secreti, unde etiam mysteria vocantur, quid valeant aenigmatum, latebrae ad amorem veritatis acuendum, discutiendumque fastidii torporem, ipsa experientia probandum est talibus, cum aliquid eis quod in promptu positum non ita movebat, enodatione allegoriae alicuius eruitur. His enim maxime utile est nosse, ita esse preaponendas verbis sententias, ut praeponitur animus corpori» (De catechizandis rudibus IX, 13). Sul ruolo cruciale svolto da Agostino in questa storia, si vedano Camper, The stylistic virtues e Antonelli, Oscurità e piacere.

10 Secondo la sintesi di Tommaso d'Aquino: «prophetia enim videtur esse quaedam cognitio obumbrata et obscuritate admixta» (Quaestio XII de veritate, a. 1; da questo brano deriva il titolo del volume di Rodolfi, Cognitio obumbrata).

11 Trachsler, Moult obscure parleüre, p. 8.

12 La teoria della profezia e le sue componenti epistemologiche sono state recentemente studiate da Rodolfi, Cognitio obumbrata; cfr. anche Torrell, Recherches e Torrell, Théorie de la prophétie. 
bile, ${ }^{13}$ ma l'influenza della psicologia araba e aristotelica accentuò ulteriormente la stretta relazione tra profezia e immagini, complice anche il fatto che nella Bibbia non esistono profezie che non passino per immagini sensibili. ${ }^{14}$

A questa riflessione teorica corrisponde un concreto stile della scrittura profetica medievale. L'oscurità dei numerosi testi pseudo-profetici elaborati tra XII e XIII secolo riposa sul presupposto che la rivelazione divina non si possa esprimere nella trasparenza del linguaggio quotidiano, e deriva dalla scrittura biblica una serie di caratteristiche spesso esasperate. Tali testi fanno ampio ricorso a un elaborato simbolismo, che viene però reso più familiare e verosimile attraverso l'impiego di metafore, già molto frequenti nelle Scritture, tratte dalla vita quotidiana - su tutte quelle animali, vegetali, meteorologiche. ${ }^{15}$ Lo schema narrativo privilegiato è quello rituale dello scontro apocalittico, che mira a suscitare una mobilitazione o un rinnovamento; ma tali testi sono sempre profondamente ambigui e malleabili, così da lasciare aperte altre possibilità future o da essere eventualmente riattualizzati qualora le profezie ivi contenute non si fossero realizzate. La loro legittimità è garantita da un semplice stratagemma di retrodatazione, che consente l'inserzione di profezie ex eventu per rafforzare la credibilità del profeta e offrire chiavi di interpretazione per le previsioni future che vi vengono avanzate.

Vediamo questi meccanismi all'opera in un esempio concreto. Tra i molti testi profetici scritti tra Due e Trecento, mi è parso particolarmente interessante il caso dell'Oraculum Cyrilli, prodotto pseudo-gioachimita probabilmente elaborato in ambito spirituale e in chiara prospettiva anti-bonifaciana nella penultima decade del XIII secolo. ${ }^{16}$ L'Oraculum godette di grande fortuna presso alcuni importanti intellettuali del Trecento: il più antico dei 26 testimoni, databile 1302, è costituito da una raccolta di scritti teologici e polemici di Arnaldo da Villanova (Roma, BAV, Borgh. 205), dove il testo - incompleto - è glossato in maniera piuttosto simile al commento pseudo-gioachimita che nella maggior parte dei manoscritti accompagna l'oracolo. ${ }^{17} \mathrm{Fu}$ poi oggetto di studio e com-

13 «Alterum, secundum spiritum, quo imaginamur ea quae per corpus sentimus; sicut vidit Petrus discum illum submitti de coelo cum variis animalibus; et sicut Isaias Dominum in sede altissima non corporaliter, sed spiritaliter vidit. Non enim Deum forma corporea circumterminat, sed quemadmodum figurate non proprie dicuntur multa, ita etiam figurate multa monstrantur» (Rabano Mauro, De universo libri XXII, PL 111, col. $72 \mathrm{a}-\mathrm{b})$.

14 Rodolfi, Cognitio obumbrata, p. 26.

15 Piron, La parole prophétique, pp. 20-1; Grévin, Rhétorique du pouvoir, pp. 213-7.

16 Sull'Oraculum Cyrilli, si vedano almeno Reeves, The influence, pp. 57-8; Piron, Allégories et dissidences, pp. 262-5; Potestà, L'ultimo messia, pp. 165-7. Il testo è pubblicato da Piur, Oraculum Angelicum Cyrilli, ma manca a oggi di un'edizione critica vera e propria.

17 Scavizzi, Abbiamo un autografo ritiene improbabile che si tratti, come a lungo creduto, della mano dello stesso Arnaldo. Sappiamo comunque che nel 1316 Arnaldo fu condannato da 
mento da parte dei francescani, che ne parlano distesamente nell'Historia septem tribulationis Ordinis minorum (1323-5), di Giovanni di Rupescissa, che attorno al 1349 vi appose un ampio commento (tràdito dal ms. Parigi, BNF, Lat. 2599), per essere poi ripreso, dopo il grande scisma, dallo pseudo-Telesforo di Cosenza e da Cola di Rienzo, che lo riassume e lo riferisce a sé in due sue epistole. ${ }^{18}$

La costruzione letteraria completa è molto raffinata: il prologo presenta il testo come ritrovato da un certo frate Gilberto inglese, «magnus theologus» non meglio identificato che racconta di aver trovato per caso nella biblioteca di Cluny un piccolo volume scritto in beneventana; l'opera vera e propria è poi preceduta da una lettera in cui un sedicente carmelitano, il presbitero Cirillo, chiede a Gioacchino da Fiore di interpretare una straordinaria rivelazione concessagli da un angelo, che l'aveva scritta su due tavole d'argento mentre Cirillo celebrava la messa. L'oracolo viene dunque spiegato dallo pseudo-Gioacchino, e l'interpretazione di questi è a sua volta commentata, in tempi successivi, da un altro autore che la inframmezzò al testo principale.

Questa complessa struttura offre qualche appiglio particolare per un confronto con le lettere dantesche: i due testi condividono non solo una cornice epistolare - certo più debole nel caso dell'Oraculum, ma comunque presente ma anche la preponderanza data all'interpretazione sulla visione. Per di più nell'Oraculum si ritrovano caratteristiche per certi versi più vicine al profetismo biblico che a quello medievale: vi mancano infatti le grandi figure escatologiche del papa angelico o dell'imperatore-messia, ma soprattutto vi è un profetizzare apocalittico piuttosto vago e debole; come Dante, che è interessato più a un'invettiva ammonitrice che a una vera previsione, l'autore dell'Oraculum lascia poco spazio a future speranze e preannuncia sventure e castighi piuttosto generici, sulla scia dei profeti vetero-testamentari. I simboli impiegati nel testo pseudo-gioachimita sono tutto sommato classici e leggibili: vi dominano figure animali che rimandano a precisi personaggi, come nella tradizione della propaganda politica italiana del XIII secolo. ${ }^{19}$ Un estratto del testo:

illis diebus frigescens Coluber reintrabit cauernam, qua ortus et alitus fuerat, simulans se velle quiescere, ubi per minutum dumtaxat quiescet. Insidiabitur autem Leoni, ut eum caluificet, et Leo insidiabitur illi, ut eius cauillam excerebret. Propter quod sicco Draconi confederabitur et sanguinee Vulpi de Anglia. Castrum quoque cum Turri fauebit eidem. Verumptamen Aries suis cornibus ventilans extendet dexteram ad Leonem, suum baiulum

un tribunale diocesano di Tarragona per aver sostenuto che l'Oraculum era da considerarsi «praetiosior cunctis Scripturis sacris» (Santi, Arnau de Vilanova, p. 286).

18 Sono le epistole 57 e 58, inviate rispettivamente all'arcivescovo di Praga e a Carlo IV di Boemia; le epistole si possono leggere in Burdach, Von Mittelalter, vol. II/3, pp. 231-332.

19 Piron, Allégories et dissidences, pp. 264-5. 
et ductorem, et aperiet illi manum, pangens cum illo, ne paueat. Sceptrum quoque versatile irrefragabiliter eidem opitulabitur tanquam suo de carne sua prudenter. Tunc Draco ille magnus, surgens de fouea, flatu adurens, et Leo ipse ferox, cauda blandiens, altercati iam dudum ad inuicem mutuo statuent duellum committere, hoc pacto, ut solus prepotens laurea fungatur post palmam. ${ }^{20}$

L'oscurità del dettato, come si vede, risiede principalmente nella difficoltà di sciogliere le equivalenze tra animali e personaggi politici; ${ }^{21}$ a tal fine il commento pseudo-gioachimita chiarisce ad esempio che il Coluber è da identificarsi con il «regnum Grecorum (...) frigescens per contumacie et obstinationis infidelitatem», e che l'azione di rientrare nella caverna, fingendo di volersi quietare, allude al suo ingannevole e temporaneo riunirsi con la Chiesa. Ancora, il leone è interpretato come reale della casa di Francia, mentre del drago il commentatore non sa offrire una spiegazione, limitandosi ad azzardare la corrispondenza con il re di Sicilia; quanto agli ultimi animali, la volpe avida di sangue rappresenterebbe il re di Inghilterra, il Castrum il re di Castiglia e l'ariete il papa. ${ }^{22}$

Metafore animali assai simili si trovano anche nelle epistole arrighiane; Dante supera però questa semplice modalità simbolica, proponendo corrispondenze più raffinate, che non mirano semplicemente a velare un personaggio politico dietro un'immagine animale ma che attirano l'attenzione su scene più complesse o su processi analogici, come ha mostrato Ledda in un suo recente articolo. Nell'epistola V compare ad esempio l'aquila, noto simbolo imperiale che però viene qui inserito in un quadro più articolato e inedito, dove si parla della cacciata dei suoi piccoli dal nido a opera di pulcini di corvo (Ep. V, 11); ancora, il «Leo fortis de tribu Iuda» $(E p . \mathrm{V}, 4)$ non è metafora che comporti una semplice identificazione con l'imperatore, ma è piuttosto una «figura di leone padre identificabile con Dio padre o forse con la divinità in generale».23 Nella missiva indirizzata a Enrico VII si ritrova invece un vero e proprio bestiario, che comprende le metafore cristologiche, riferite all'imperatore e a suo figlio, del leone e dell'agnello ( $E p$. VII, 5) e le trasfigurazioni di Firenze in idra (Ep. VII, 20), «vulpecula», vipera e pecora infetta (Ep. VII, 23-6); la semplice equivalenza

20 Piur, Oraculum Angelicum Cyrilli, pp. 269-70.

21 Da notare che «nei testi sibillini e oracolari, gli animali simbolici rappresentano una costante, utile ad avallare la pretesa origine soprannaturale dei testi e la loro ambigua cifra veritativa. Gli elementi ritenuti propri di ciascun animale sono ricavati dalla scienza dei bestiari, le cui nozioni per la decifrazione dei significati simbolici risultano però continuamente riadattate in funzione di vicende, popoli e singoli personaggi» (Potestà, L’ultimo messia, p. 85). Sull'uso delle metafore animali nella propaganda politica cfr. anche Lerner, Ornithological propaganda.

22 Piur, Oraculum Angelicum Cyrilli, pp. 270-1.

23 Ledda, Un bestiario politico, pp. 162-9. 
tra res e signum della comunicazione profetica viene annullata nel momento in cui a uno stesso referente rimandano immagini tanto diverse.

\section{La transumptio nell'ars dictaminis}

Lo stesso patrimonio di immagini bibliche era ampiamente ripreso dai dictatores, professionisti della retorica cancelleresca che ambivano a sacralizzare la propria attività ponendosi non solo come maestri di una tecnica di scrittura, ma anche come depositari di una sapienza esegetica che permetteva loro di manipolare l'interpretazione dei testi normalmente affidata alla Chiesa. ${ }^{24}$ Le epistole emanate dalle cancellerie papale e imperiale durante l'acceso scontro tra Federico II e Gregorio IX si appropriarono di una retorica profetico-apocalittica che da entrambe le parti mirava a demonizzare l'avversario, presentandolo come l'Anticristo preannunciato dalle Scritture. Nello stesso periodo una rinnovata attenzione nei confronti dell'esegesi e delle possibili riattualizzazioni di simboli polisemi contribuì ad alimentare una scrittura oscura, allusiva e densa di elementi simbolici, sfida ermeneutica e vero e proprio argot di un'élite intellettuale. Poiché l'essenza dell'argot è quella di essere un signum, una modalità discorsiva che distingue il parlante e il suo gruppo dagli altri locutori, l'ingresso di tale linguaggio segreto all'interno del discorso pubblico può comportare la sua conservazione come segno stilistico; ${ }^{25}$ in questo modo un codice misterioso come quello profetico, adottato in un altro contesto, perse buona parte della sua carica di oscurità, rimanendo semplice marcatore di un linguaggio esclusivo.

La pragmatica del linguaggio figurato teorizzato e impiegato nell'ars dictaminis è perciò differente, ma non del tutto, rispetto a quella propria del profetismo medievale: in entrambi i casi la ricerca di un dettato oscuro contribuisce alla credibilità e alla ieraticità del testo, e in entrambi i casi l'autore rivendica la propria capacità di dominare i simboli tanto dal punto di vista produttivo quanto da quello interpretativo. Ma mentre gli autori di testi profetici avevano bisogno di un discorso che riuscisse a rimanere sufficientemente ambiguo e malleabile da poter essere riadattato in tempi successivi, i dictatores che scrivevano per conto di una cancelleria avevano come obiettivo principale la comprensibilità e l'incisività del messaggio.

Ai testi dell'ars dictaminis è stata a lungo conferita una patente di oscurità che sembra debba essere meglio contestualizzata: secondo Witt, lo stilus rhetori-

24 Sulle strategie di legittimazione messe in campo dai dictatores, si vedano almeno Artifoni, Sapientia Salomonis e Grévin, Métaphore et vérité.

25 Si tratta del «transfert de la fonction sémantique» di cui parla Guiraud, L'argot, pp. 102-3. 
cus sviluppato dalle cancellerie sotto l'influenza dei sermoni sulle crociate, e perciò ricco di citazioni bibliche e classiche, non coincide del tutto con lo stilus obscurus che i dictatores usavano nelle proprie missive private, dove i colleghiamici venivano sfidati attraverso un linguaggio da casta che faceva continuo sfoggio di bravura e che permetteva di sfuggire dalla routine di una scrittura formulare. ${ }^{26}$ Grévin ha invece messo in discussione la distinzione proposta da Witt e ribadito che la contraddizione tra oscurità ed esigenze pragmatiche della comunicazione imperiale è solo apparente, dal momento che tale oscurità promuove una sacralizzazione quasi magica del linguaggio; la distinzione sembrerebbe essere, semmai, tra i dictatores della curia, che sostengono una certa chiarezza linguistica, e quelli imperiali, che non esitano a ricorrere alla transumptio anche a rischio di scadere nell'oscurità. ${ }^{27}$

Il discorso tocca soprattutto Pier della Vigna, maestro indiscusso dell'epistolografia latina che proprio per la sua oscurità era noto ai contemporanei, se accettiamo la testimonianza di Odofredo: «volentes obscure loqui et in supremo stilo, ut faciunt summi doctores et sicut faciebat Petrus de Vineis». Al logoteta di Federico II si può attribuire un'eccezionale conoscenza di Scritture e glosse, che gli permise di sviluppare all'interno della cancelleria sveva il raffinatissimo stile elaborato dalla Curia papale sotto Innocenzo III. L'impiego, «con intenti ideologici, persuasivi e polemici del linguaggio biblico» genera un dettato non sempre comprensibile, volto a «creare il senso di una oscura minaccia»; ${ }^{28}$ lo caratterizzano un periodo sintatticamente assai complesso, un ampio ricorso alla citazione classica e scritturale, una sostenuta tensione che non sfocia mai nella violenza di altri documenti della coeva propaganda politica, ma che non rifugge dal sarcasmo o da severe invettive. ${ }^{29}$

Il confronto tra la prosa di Pier della Vigna e quella dantesca non è una novità: lo stesso Baglio, curatore del più recente commento alle epistole di Dante, inquadra la produzione di quest'ultimo all'interno degli schemi dell'ars dictaminis e propone numerosi paralleli con le missive della cancelleria sveva. ${ }^{30}$

26 Witt, Medieval ars dictaminis, pp. 14-5.

27 Sull'obscuritas in relazione al dictamen, si vedano Grévin, Rhétorique du pouvoir, in part. p. 205 e 259 e Grévin, «Linguistic mysteries».

28 Boccia, Forme della creazione, pp. 87-88.

29 Sullo stile di Pier della Vigna, si vedano Mazzamuto, L'epistolario; Grévin, Rhétorique du pouvoir; l'introduzione di L'epistolario di Pier della Vigna; Delle Donne, Le parole del potere, in part. pp. 121-122.

30 Si veda in particolare Ep. (Baglio), p. 24. Altri raffronti tra Pier della Vigna e Dante sono proposti, in questo stesso volume, dal saggio di Benoît Grévin, che se ne era già occupato in Grévin, Rhétorique du pouvoir, pp. 795-7; discute il rapporto di Dante con il dictamen Montefusco, Le "Epistole"; sul rapporto tra Dante e Pier della Vigna si veda anche Montefusco, Pier della Vigna. L'articolo dello stesso Montefusco in questo stesso volume (Competenze, prassi e 
Dalla specola del linguaggio figurato, risulta però particolarmente interessante rivedere qualche passaggio di una delle più celebri epistole di Pier della Vigna, la Collegerunt pontifices che apre la raccolta epistolare tràdita sotto il suo nome. ${ }^{31}$ Questa missiva, secondo i curatori della raccolta, è esemplare tanto a livello politico, quanto a livello retorico, perché pur abbracciando i modi accesissimi della polemica in corso li traduce in una forma nuova, dove il registro biblico-apocalittico si alterna a quello satirico-antifrastico: ${ }^{32}$

vineam autem Domini Sabaoth aliis locabit agricolis et bonos absque iudicio male perdet. Obstemus ergo principiis, ne forte scintilla tenuis in flammam transeat destructiuam, et morbus quidem sic fistulare incipiens, non abscissus perueniat in medullas. (...) Ploret igitur mater Ecclesia, quod pastor gregis Dominici factus est lupus rapax! Crassum quidem de grege comedens, confractum non alligat, et dissolutum ad propria non reducit, sed uelut amator schismatis, caput et actor scandali, pater doli, contra iura Romani principis et honorem tuetur hereticos, Dei quidem et omnium Christi fidelium inimicos, omni prorsus Altissimi timore postposito et hominum uerecundia ultroiecta. (...) Reuertentem ergo ad gremium matris Ecclesiae benigne recipias filium singularem, presertim cum petat instanter ueniam sine culpa: aloquin leo noster fortissimus, qui hodie simulat se dormire, rugitu solo terribili trahet omnes a terrae finibus tauros pingues, et plantando iustitiam, Ecclesiam diriget, euellens prorsus et destruens cornua superborum. ${ }^{33}$

Come si vede, l'elaborazione metaforica è sostenuta e articolata in scene di una certa estensione, che riprendono immagini naturali tratte dalla Bibbia (la vigna del Signore, la madre Chiesa, il pastore fatto lupo rapace e via dicendo); la prosa è difficile ma non completamente oscura, grazie al riferimento a brani riconoscibili e a un andamento ragionativo comprensibile, per quanto impegnativo. La solennità non scade in un'eccessiva ridondanza, come accade in tanti altri brani dettatori: ${ }^{34} \mathrm{i}$ traslati animano il dettato in maniera dinamica e concre-

legittimità profetica del Dante dictator illustris) affronta nuovamente il tema, argomentando contro un'eccessiva riduzione dello stile epistolare di Dante all'influenza di Pier della Vigna. 31 Sulla Collegerunt pontifices, si veda anche Falzone, Fiorentini, Note sul discorso, pp. 21721.

32 L'epistolario di Pier della Vigna, pp. 56-62.

33 L'epistolario di Pier della Vigna, pp. 79-82 (PdV I, 1, parr. 2; 18; 33).

34 Un breve esempio, di passaggio, può essere offerto dalla ripetitiva metaforica della bolla Exultet in gloria, con cui Clemente V salutava l'elezione di Enrico VII (1 settembre 1310): «hec est enim fructus benedictus a Domino, per quem mellifluam celi dulcedinem susceperunt. Hec est fructus pretiosus fidelibus, quem arbor iustitie in benedictionibus plantata dulcedinis germinavit. Hec est fructus mire pulcritudinis et decoris, quem celestis agricole summa benignitas de radice caritatis eduxit. Et hec est fructus, de quo gentium doctor apostolus et predicator egregius veritatis in sacro sue salutationis eloquio, quod de conscientia regis emittebat excelsi, cunctorum refici corda fidelium cum summo desiderio cupiebat» (Henrici VII Constitutiones, p. 376). 
ta, le personificazioni diluiscono l'estraneità delle metafore, ma l'ossequio ai dettami dell'amplificatio si fa sentire nell'estensione non sempre necessaria di un tema. Le metafore animali, in particolare, sono assimilabili non solo ai precedenti scritturali, quanto a certi modi della letteratura profetica medievale; rispetto all'esempio dell'Oraculum, però, il dettato di Pier della Vigna non si limita a una semplice trasfigurazione zoomorfa, ma realizza un più complesso gioco di alternanze tra auctoritates, segmenti ragionativi, narrativi, polemici. L'apparato metaforico elaborato dal dictator svevo risulta così un precedente da tenere presente nell'analisi delle epistole arrighiane, che pure non lo imitarono in maniera pedissequa e si spinsero ben più in là nel rifiutare una retorica meramente esornativa e nel riconoscere alle citazioni e all'imagery un ruolo argomentativo e persuasivo dominante.

Pier della Vigna sembra tradurre in pratica un pensiero della transumptio che si era andato parallelamente sviluppando in ambito teorico per iniziativa dei dictatores attivi a Bologna nella prima metà del XIII secolo. Maestri come Boncompagno da Signa, Bene da Firenze e Guido Faba reagirono al predominio della scuola d'Orléans e del suo lessico eccessivamente ricercato e propugnarono una retorica egemonica rispetto agli auctores pagani, pensata per i bisogni pratici del ceto notarile; la dottrina della transumptio così elaborata recuperava alcuni aspetti esegetici provenienti dalla teologia chartriana e dagli insegnamenti delle artes poetriae nell'ottica di una scrittura sì sacralizzante, ma comprensibile e adatta alla situazione comunicativa, dove solo sul principio inderogabile della correttezza si poteva innestare un procedimento di ornamento stilistico.

Riprendendo la Poetria nova di Goffredo di Vinsauf, i dictatores assegnarono all'ornatus difficilis i dieci tropi enucleati dalla Rhetorica ad Herennium e li riassunsero sotto il nome di transumptio. ${ }^{35}$ Il Candelabrum di Bene, in particolare, sciolse la confusione operata da Goffredo tra transumptio e translatio e considerò la prima un generico modo del parlare figurato tendente alla gravitas, a cui appartiene la seconda, semplice tropo equivalente a quella che oggi chiamiamo metafora. La transumptio, per Bene, si realizza infatti soprattutto in quattro dei dieci tropi della tradizione: nominatio, pronominatio, permutatio, translatio (VII, xxviii, 2-3). Il primo corrisponde all'onomatopea; gli altri tre tropi alla base della transumptio sono definiti in questo modo:

pronominatio est que velut extraneo quodam vocabulo sive cognomine demostrat id quod suo non potest nomine appellari (...). Permutatio fit in nomine tam proprio quam comuni.

35 Sulla transumptio cfr. Forti, La "transumptio”; Purcell, Transsumptio; Grévin, Métaphore et vérité; sulla transumptio nelle artes poetriae si veda Tomazzoli, Nova quaedam. 
Hec aliud verbis, aliud sententia, sicut allegoria, demostrat et fit per similitudine, argumentum atque contrarium (...). Traslatio est ex quadam convenienti similitudine verbi iam dudum inventi in aliam rem traductio. Hec fit tam in nomine quam in verbo et partipicio et adverbio et in hoc differt a precedentibus. In permutatione tamen significatio vocabuli non mutatur sed per rem ipsius alia res intelligitur (...) In translatione vero dictio quasi sponte ad aliud significandum convertitur (VII, xxiv-xxxii).

\section{La transumptio e l'explanatio nelle epistole arrighiane}

Come prescritto da queste norme relative alla gravitas, i tre tropi dell'antonomasia, dell'allegoria e della metafora sono abbondantemente presenti nelle epistole arrighiane di Dante. A uno spoglio sistematico i termini figurati risultano coprire una discreta parte della prosa dantesca, pur addensandosi in maniera diseguale nel corpo delle tre epistole.

Spicca soprattutto l'ampio ricorso alla pronominatio, con espressioni come il già citato «Leo fortis de tribu Iuda» (Ep. V, 4), ma anche «Moysen alium» (Ep. V, 4), «tanquam alteri Babilonii» (Ep. VI, 6), «Pergama rediviva» (Ep. VI, 15), «Goliam hunc» (Ep. VII, 2), «nobis est alter Ascanius» (Ep. VII, 18), «hec Myrrha scelestis et impia», «hec Amata illa impatiens» (Ep. VII, 24), «proles altera Isai» (Ep. VII, 29). La strategia della pronominatio comporta la designazione, attraverso un nome proprio, di un modello o di un insieme di caratteristiche; insieme alla metafora, l'epiteto è il modo per eccellenza di una comunicazione criptata, dove il linguaggio segreto agisce attraverso una sostituzione e dove la decifrazione dipende interamente dalla conoscenza autonoma, da parte del lettore, delle caratteristiche dell'archetipo. ${ }^{36}$ La pronominatio risulta perciò una figura ideale per realizzare un tono solennemente profetico, perché grazie a essa eventi della storia contemporanea vengono messi in relazione con il grande quadro storico-provvidenziale che si distende a partire dal passato del mito classico e delle Scritture.

Consistenti anche le permutationes, ossia le allegorie. Un esempio caratteristico della scrittura dantesca è l'immagine del campo della mente che compare nell'epistola V:

assumite rastrum bone humilitatis, atque glebis exuste animositatis occatis, agellum sternite mentis vestre, ne forte celestis imber, sementem vestram ante iactum preveniens, in vacuum de altissimo cadat. Non resiliat gratia Dei ex vobis tanquam ros quotidianus ex

36 Guiraud, L'argot, p. 54. 
lapide; sed velut fecunda vallis concipite ac viride germinetis, viride dico fructiferum vere pacis; qua quidem viriditate vestra terra vernante, novus agricola Romanorum consilii sui boves ad aratrum affectuosius et confidentius coniugabit (Ep. V, 15-6).

Il passo riprende motivi scritturali e patristici, alcuni dei quali già impiegati nella propaganda papale e imperiale, ma li armonizza in un'unica grande immagine, dove diverse metafore concorrono a creare un piccolo universo psicologico, coeso e delineato in toni di fervente coinvolgimento spirituale e intellettuale.

Il tropo assolutamente dominante è però la translatio, che viene impiegata nei modi più diversi. A catene semantiche coerenti si avvicendano immagini in forte contrasto; si alternano metafore topiche e originali, brevi ed estese, naturalistiche e letterarie.

A, Tuscorum vanissimi, tam natura quam vitio insensati! Quam in noctis tenebris malesane mentis pedes oberrent ante oculos pennatorum, nec perpenditis nec figuratis ignari. Vident namque vos pennati et inmaculati in via, quasi stantes in limine carceris, et miserantem quempiam, ne forte vos liberet captivatos et in compedibus astrictos et manicis, propulsantes (Ep. VI, 21).

Questo estratto è un vero tour de force di metafore: in un ritmo incalzante le tenebre dell'ignoranza e del peccato si legano non agli attesi occhi interiori, ma agli stranianti pedes della mente, che vengono poi messi a contrasto con l'exemplum degli uccelli; a loro volta questi sono accostati, con ulteriore traslazione, agli uomini inmaculati, ossia innocenti, prima che prenda il sopravvento l'ultima metafora estesa del carcere. La stessa sovrapposizione di immagini si verifica in quest'altro passaggio, dove la metafora della pecora malata già introdotta si intreccia con la ripresa della pronominatio di Mirra, che nel suo ruolo di seduttrice del padre attira l'epiteto papale di pater patrum:

vere fumos, evaporante sanie, vitiantes exhalat, et inde vicine pecudes et inscie contabescunt, dum falsis illiciendo blanditiis et figmentis aggregat sibi finitimos et infatuat aggregatos. Vere in paternos ardet ipsa concubitus, dum improba procacitate conatur summi Pontificis, qui pater est patrum, adversum te violare assensum (Ep. VII, 26).

In tanta densità e complessità metaforica, spicca una caratteristica significativa: la pressoché totale mancanza di oscurità; per quanto il dettato possa essere elaborato o i riferimenti impegnativi, Dante si preoccupa sempre della comprensibilità, oltre che della solennità, del suo discorso. Questa preoccupazione è particolarmente evidente nel caso di alcune precisazioni che giungono a chiarire o dichiarare il tropo. È quel che accade ad esempio in questo passaggio: «absit, quoniam Augustus est. Et si Augustus, nonne relapsorum facinora vindicabit, et usque in Thessaliam persequetur, Thessaliam, inquam, finalis deletio- 
nis?» (Ep. V, 10). L'inciso finale conferisce ulteriore gravità alla già sostenutissima interrogativa retorica, e la dichiarazione dell'immagine veicolata dalla nominatio non stempera il quadro ma lo estende: il riferimento storico alla Tessaglia, avvolto in una distanza letteraria classicheggiante, da archetipo di sconfitta si innalza a scenario apocalittico di distruzione.

Un altro esempio è contenuto all'interno del passo appena citato, dove la complessa metafora vegetale si intreccia con le immagini bibliche della pioggia e della rugiada; la tensione si scioglie nel dolce e allitterante sintagma finale, che arriva a coronare e risolvere l'ampio quadro sviluppato: «sed velut fecunda vallis concipite ac viride germinetis, viride dico fructiferum vere pacis» (Ep. V, 16). Questa prassi sembra corrispondere a quel procedimento che i retori medievali, sulla scorta della Rhetorica ad Herennium, ${ }^{37}$ chiamavano explanatio: è il principio opposto all'obscuritas, nonché uno dei due pilastri di un discorso elegante e perspicuo. Lo riassume ancora una volta in maniera esemplare il Candelabrum:

sed non est satis loqui congrue vel latine, quia posset ibi esse rerum impertinentia vel inconcinna translatio vel obscuritas vitiosa, et ideo necessaria est explanatio ad elegantiam obtinendam, quia nichil est oratori convenientius quam conceptam materiam verbis idoneis explanare (...) Sciendum est quod explanationem inducunt usitata verba et propria seu competenter aliunde translata. ${ }^{38}$

L'explanatio offre dunque una via d'uscita dagli angusti perimetri della semplice latinitas perché permette di introdurre nel discorso anche dei termini "inusitati" o "impropri", purché la traslazione sia padroneggiata con competenza e cognizione di causa.

\section{Analogie argomentative: un'epistemologia dei segni}

Del resto il concetto stesso di oscurità esiste in mutua dipendenza con il suo opposto, la chiarezza. L'oscurità è il presupposto di ogni atto esegetico, perché

37 «Explanatio est quae reddit apertam et dilucidam orationem. Ea conparatur duabus rebus, usitatis verbis et propriis. Usitata sunt ea quae versantur in consuetudine cotidiana: propria, quae eius rei verba sunt aut esse possunt qua de loquemur» (Ad Her. IV, xii, 17).

38 Bene da Firenze, Candelabrum, I, xi, 10-1; xii, 1-7; questo passo è commentato in Montefusco, Bischetti, Prime osservazioni, pp. 201-203. Anche un altro dictator come Riccardo da Pofi ribadiva la necessità di illustrare chiaramente anche le epistole che si avvalgono di auctoritates bibliche o profetiche: «ces remarques montrent que les dictatores étaient pleinement con- 
non sarebbe necessario spiegare un testo già comprensibile: la sua eliminazione sembra perciò essere l'obiettivo di ogni interpretazione. Il procedimento dantesco risolve l'oscurità nel momento in cui la crea, semina indizi e poi li spiega, impiega citazioni e immagini che vengono poi meglio contestualizzate. Buona parte della carriera di Dante è segnata dal rifiuto di un'oscurità dettata da imperizia, da superbia o da spirito settario, a favore di una difficoltà dipendente dall'inerente nobiltà del contenuto ma sempre comprensibile; prova ne sono la polemica con Guittone, ${ }^{39}$ ma anche i diversi passi della Commedia in cui il linguaggio delle profezie è opposto a quello chiaro. ${ }^{40}$

Le forme di oscurità che operavano nella scrittura profetica da un lato e in quella dettatoria dall'altro non hanno ragione di esistere nel caso dantesco: a differenza dei dictatores, Dante non parla per conto di un'istituzione, e a differenza degli autori di testi profetici la legittimazione che egli cerca è soprattutto intellettuale e morale. ${ }^{41}$ Se profezia e dictamen sono due modalità diverse di parlare di una politica altamente retoricizzata attraverso una retorica politicizzata, le epistole arrighiane intraprendono un altro percorso, dove l'invettiva profetizzante non può prescindere dalla carica persuasiva dell'ars e dove quest'ultima non è mai fine a sé stessa. La postura assunta da Dante in questi testi implica il doppio momento dell'interpretazione e della predicazione: l'autore fonda l'autorevolezza del proprio discorso su una corretta comprensione dei segni offerti dalla realtà, e al contempo proclama quanto ha appreso mediandolo attraverso analogie, citazioni ed exempla che hanno il duplice scopo di chiarire e di persuadere.

Come sostiene Ledda, quello che Dante adotta nelle epistole V e VI è un «profetismo naturale e argomentativo»: il primo dei due testi annuncia in tono trionfante la venuta di Enrico, celebrata attraverso scene di rinascita ispirate alle Scritture e alla classicità, e giustifica tali riprese con un «brano che si po-

scients du danger que présentait la volonté d'assimiler la dignité du discours politique à celle $\mathrm{du}$ langage biblique en donnant au premier, par l'importation d'un langage allégorique, la dignité du second» (Grévin, «Linguistic mysteries»).

39 Sull'obscuritas guittoniana e sul suo rapporto con il dictamen, si veda Montefusco, La linea, pp. 5-10.

40 Come si vede bene nel discorso di Cacciaguida, dove le chiare parole e il preciso latin (forse non dimentico della latinitas retorica) si oppongono alle ambage folli dei pagani: «né per ambage, in che la gente folle / già s'inviscava pria che fosse anciso / l'Agnel di Dio che le peccata tolle, / ma per chiare parole e con preciso / latin rispuose quello amor paterno, / chiuso e parvente del suo proprio riso» (Par. XVII, 31-6).

41 Un discorso parzialmente diverso si apre per l'Ep. XI, dove la postura profetica assunta da Dante rimanda piuttosto a modelli vetero-testamentari, e dunque a un'investitura carismatica; cfr., in proposito, i due articoli di Montefusco e Potestà in questo volume. 
trebbe definire metaprofetico». ${ }^{42} \mathrm{I}$ «signa (...) consolationis et pacis» $(E p . \mathrm{V}, 2)$ si realizzano in gioiose immagini che manifestano la provvidenzialità della missione arrighiana: sono le topiche metafore dell'alba opposta alle tenebre (Ep. V, 2), del sole della giustizia ( $E p . \mathrm{V}, 3)$, dell'imperatore come sposo (Ep. V, 5), della valle feconda in cui germoglia la pace (Ep. V, 15-6) - per citarne solo alcune. Nella parte finale dell'epistola queste metafore vengono inquadrate non come dispositivi retorici messi in campo da Dante, ma come meravigliosi effetti della volontà di Dio, testimoniati dalla Chiesa e confermati dal Papa (Ep. V, 22). Contaminando San Paolo con Aristotele, Dante spiega che gli invisibilia di Dio si comprendono attraverso le creature, e che le cose sconosciute ci si rivelano attraverso quelle conosciute $(E p . \mathrm{V}, 23) .{ }^{43} \mathrm{~A}$ questi argomenti razionali subentrano argomenti storico-provvidenziali (Ep. V, 26-9), infine suggellati dall'auctoritas papale (Ep. V, 30).

Questa operazione interpretativa viene poi rovesciata nell'epistola seguente, che comincia con il dichiarare le disposizioni della provvidenza divina: ai «divinis elogis», confermati dalla ragione e dalla storia (Ep. VI, 2-3), Dante oppone la tracotante e cieca ribellione dei Fiorentini, che si realizza in un'immaginosa galleria di mali futuri ( $E p$. VI, 5-16). L'invettiva viene anche in questo caso giustificata razionalmente:

et si presaga mens mea non fallitur, sic signis veridicis sicut inexpugnabilibus argumentis instructa prenuntians, urbem diutino merore confectam in manus alienorum tradi finaliter, plurima vestri parte seu nece seu captivitate deperdita, perpessuri exilium pauci cum fletu cernetis (Ep. VI, 17).

Dante non afferma qui di aver ricevuto un'investitura carismatica, ma di aver saputo interpretare i segni che la Provvidenza ha impresso sulla storia: i vasti quadri metaforici disegnati nelle epistole, nel patto testuale, non sono altro che figure della realtà, le uniche a essere comprensibili nell'insondabile mistero divino e le più efficaci per trasmettere la parola profetica. Per questo le epistole

42 Ledda, Modelli biblici, pp. 24-8.

43 Lo stesso brano paolino ( $R m$ 1, 20) viene citato nella Monarchia, in una dimostrazione, cruciale per l'impalcatura teorica del trattato, sulla conformità del diritto umano alla volontà divina: «preterea meminisse oportet quod, ut Phylosophus docet in primis ad Nicomacum, non similiter in omni materia certitudo querenda est, sed secundum quod natura rei subiecte recipit. Propter quod sufficienter argumenta sub invento principio procedent, si ex manifestis signis atque sapientum autoritatibus ius illius populi gloriosi queratur. Voluntas quidem Dei per se invisibilis est; et invisibilia Dei "per ea que facta sunt intellecta conspiciuntur"; nam, occulto existente sigillo, cera impressa de illo quamvis occulto tradit notitiam manifestam. Nec mirum si divina voluntas per signa querenda est, cum etiam humana extra volentem non aliter quam per signa cernatur» (Mon. II, ii, 7.8). 
arrighiane impiegano le auctoritates non in semplice alternanza con la ratio, come prescritto dalle artes dictandi e come sperimentato dalla prosa epistolare di Guittone, ma come scaturigini di campi di immagini funzionali a un'argomentazione razionale e icastica. A tal scopo Dante non può avvalersi delle metafore generiche e simboliche della scrittura profetica, basate su una semplice identificazione tra res e verba, né delle metafore dettatorie, che impreziosiscono il documento secondo i modi dell'amplificatio; a tal scopo servono infatti metafore che mettano a fuoco un processo o un'ampia immagine, illuminando il dipanarsi della storia e mostrando lo schema razionale dietro agli eventi. Questo parlare figurato crea l'illusione dell'oscurità solo per spiegarla: quella che formalmente è perfetta adesione di forma e contenuto, epistemologicamente rimanda tanto alla sfida ermeneutica della decifrazione delle immagini quanto all'unico linguaggio efficace per veicolare questa interpretazione. 
